\title{
INVESTIGATION OF APS PAR VERTICAL BEAM INSTABILITY *
}

\author{
CY Yao, Y. Chae, N. S. Sereno and B. Yang \\ Advanced Photon Source, Argonne National Laboratory, Argonne, IL 60439, U.S.A.
}

\begin{abstract}
A vertical beam instability has been observed for some time in the particle accumulator ring (PAR) of APS. It appears at low beam current when a single linac bunch is selected for PAR injection and is highly reproducible. An investigation was conducted to characterize and understand this instability. We obtained some interesting data and believe this was due to ion trapping. A more stable lattice was established as result of the investigation. This report summarizes the experimental results and gives some preliminary analysis.
\end{abstract}

\section{INTRODUCTION}

The particle accumulator ring (PAR) is an accumulator ring that captures multiple linac bunches into a single fundamental rf (RF1) bucket and then compresses the bunch with a $12^{\text {th }}$ harmonic $\mathrm{rf}$ (RF12) to a length of $0.3 \mathrm{~ns}$ for booster synchrotron injection. The cycle time of the PAR is $500 \mathrm{~ms}$.

A vertical instability is observed when a single linac bunch is selected for PAR injection and the charge of bunch is between $0.1 \mathrm{nC}$ to $0.7 \mathrm{nC}$. The instability stabilizes itself in the latter part of the PAR cycle, about $300 \mathrm{~ms}$ after the injection. In stored-beam mode, pulsing of the kicker magnets is needed to induce the instability. The data we obtained indicate that the instability is due to ion trapping. This report summarizes the experimental results and preliminary analysis.

\section{EXPERIMENT SETUP}

The PAR was set up for either continuous injection mode or stored mode with a charge of $0.1 \mathrm{nC}$ to $0.9 \mathrm{nC}$. In stored-beam mode, extraction and the linac beam were disabled but the injection kickers were still fired. A scraper in the linac was used to adjust the charge injected into the PAR to any desired value.

An HP vector signal analyzer (VSA) was used to observe and record the beam spectrum. A timing signal derived from the injector timing system served as external trigger to the VSA. The VSA can be operated in single, average and time-capture modes.

A synchrotron radiation monitor was used to visually observe the beam transverse profile. A CCD camera and a gated camera were used to capture images of the beam transverse profile.

\section{OBSERVATIONS AND RESULTS}

With PAR injection with one linac bunch, we were able to reproduce the vertical instability easily. The instability

\footnotetext{
*Work supported by U.S. Department of Energy, Office of Basic

Energy Sciences, under Contract No. W-31-109-ENG-38.
}

was clearly a vertical oscillation with the core of the bunch bouncing, as seen on the synchrotron radiation monitor.

\section{Accumulated Spectrum}

Figure 1 shows spectra accumulated over many injection cycles at different beam charges. At $0.1 \mathrm{nC}$, beam is stable and the vertical spectrum showed a single tune spike. As the charge increased, more spikes appeared and the width of each spike increased. At $0.5 \mathrm{nC}$, the instability as seen on the synchrotron radiation monitor became more severe and the spectrum spikes overlapped and formed a plateau shape. At $0.8 \mathrm{nC}$, the instability became intermittent. Above $0.8 \mathrm{nC}$ beam became stable again. The appearance of each spectrum spike was in coincidence with vertical bounces of the beam spot on the synchrotron radiation monitor. Although the occurrence of individual spikes appeared random, the lower frequency edge of the spectrum matched to the vertical tune of a stable beam. Another character is that the total width of the plateau shape did not seem to vary with beam current.

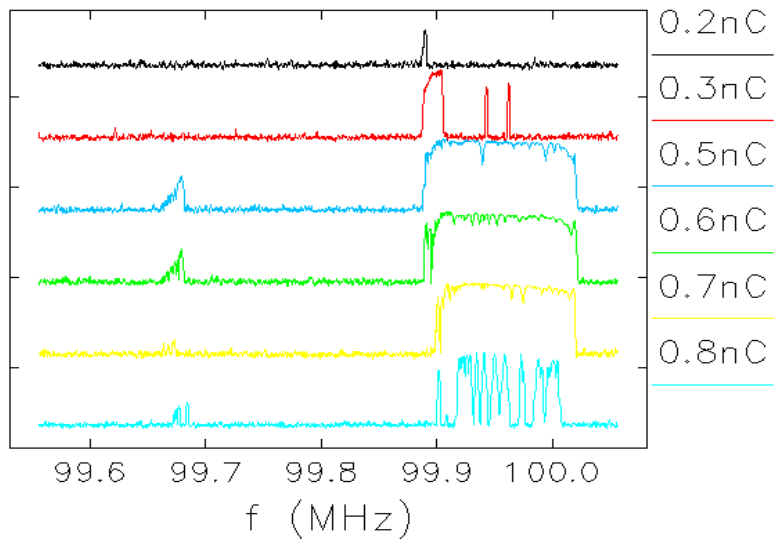

Figure 1: Accumulated beam spectra for different beam charge. Vertical is staggered and the spacing is $40 \mathrm{dBm}$. Notice the vertical tune waveforms are the broad peaks on the right while the horizontal tune waveforms are the narrow peaks on the left.

\section{Transverse Profile}

Figure 2 shows plots of the images from the CCD camera. Apparently there was a centroid beam oscillation. The gated-intensified camera, which captured the beam image in a single pass, showed a bouncing motion with the core of the bunch moving up down and sometimes also changing its shape. This indicated the existence of coherent beam motion.

\section{Timing Character}

Figure 3 is a diagram of a PAR cycle for timing reference. The kickers are fired once for each linac bucket 
selected for injection. The interval between linac buckets is $33 \mathrm{~ms}$. RF12 is turned on 250 to $400 \mathrm{~ms}$ after first injection.

We noticed that the instability stopped when two or more linac buckets were selected for PAR injection. Scanning delay time of the gated camera, we found that the instability actually started about $80 \mathrm{~ms}$ after injection and lasted $\sim 180 \mathrm{~ms}$.
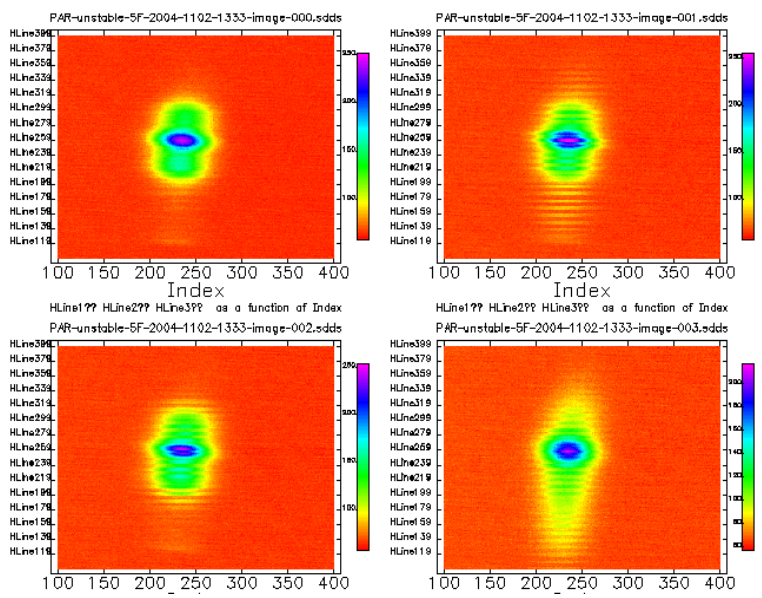

Figure 2: Multi-turn beam profile CCD camera image. Each frame represents an integration time of $33 \mathrm{~ms}$.
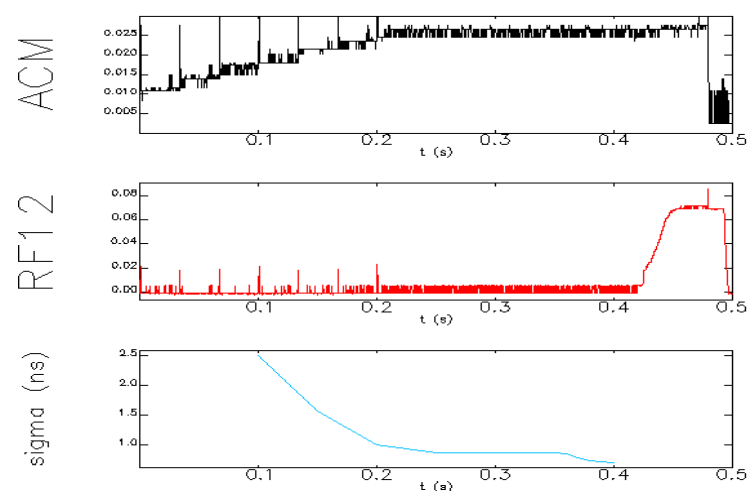

Figure 3: Timing relation of a PAR injection cycle. The top trace is the PAR current with seven linac buckets for injection. The middle trace is the RF12 waveform. The bottom trace is bunch-length measurement result.

This timing character was further studied in storedbeam mode. Beam was stable without firing the kickers. Instability appeared when kickers were fired once per cycle. In this case the instability started right after the firing of the kickers. Firing the kickers two or more times per cycle caused the instability to stop. We concluded that a perturbation from a single kicker pulse was necessary to introduce the instability and additional kicker firing disrupted the instability.

Figure 4 shows time-captured data of the VSA while the PAR was in injection mode, which provides a better picture of the timing character of the instability. The instability spectrum trace started $70 \mathrm{~ms}$ after a kicker pulse, which is indicated by the straight horizontal trace. The vertical oscillation frequency shifted up by about 200 $\mathrm{kHz}$ in about $20 \mathrm{~ms}$. It remained at the frequency for about $60 \mathrm{~ms}$ and then shifted back toward normal vertical tune. The total process lasted up to $180 \mathrm{~ms}$.

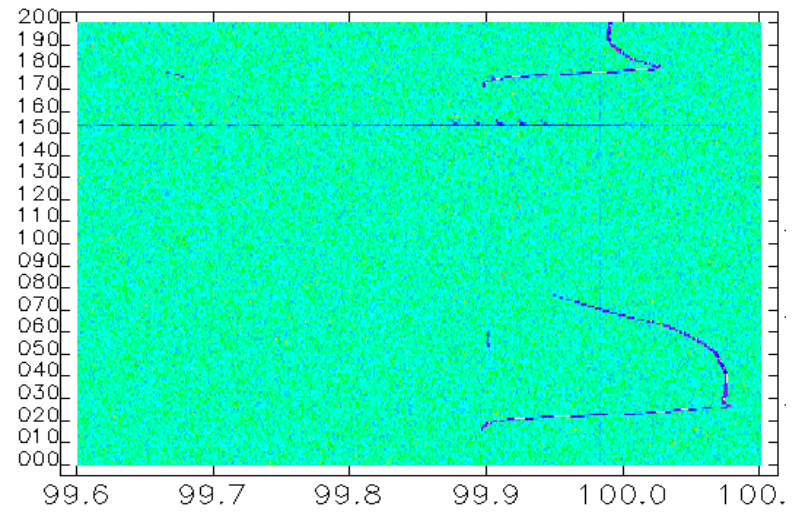

Figure 4: Time-captured spectrum of unstable beam. The vertical axis is time in unit of $3.2 \mathrm{~ms}$, while the horizontal axis is frequency in $\mathrm{MHz}$.

Figure 5 shows another interesting feature of the instability. Two of the instability traces actually overlap each other for a period. On the synchrotron monitor this corresponded to double bouncing of the beam in the vertical plane.

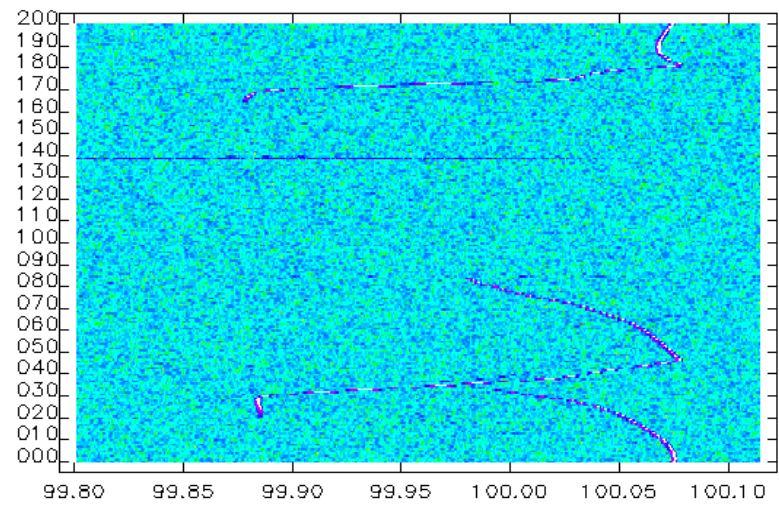

Figure 5: Unstable beam spectra, showing overlapping of two unstable traces.

\section{COHERENT ION TRAPPING}

From our preliminary investigation, we believe that this is a coherent ion trapping instability. Its current dependency is different from an impedance-driven instability. The latter typically has a current threshold above which beam is unstable.

Ion trapping instabilities have been studied and reported by many authors [1-4]. The criterion for ion trapping is expressed as critical mass:

$$
A>A_{c}=N_{e} \frac{L r_{p}}{2 \sigma_{y}\left(\sigma_{x}+\sigma_{y}\right)},
$$


where $\mathrm{N}_{\mathrm{e}}$ is the total number of electrons in the bunch, $\mathrm{r}_{\mathrm{p}}$ is the classic proton radius, $\mathrm{L}$ is the circumference of the ring, $A$ is the mass of an ion, $A_{c}$ is the critical massnumber, and $\sigma_{\mathrm{x}}$ and $\sigma_{\mathrm{y}}$ are the horizontal and vertical beam sizes, respectively.

Only ions with mass higher than $A_{c}$ can be trapped. In the PAR case any ions with a mass higher than 2 can be trapped.

A trapped ion oscillates with a frequency described by the following expression [5]:

$$
f_{i}=\frac{c}{2 \pi} \sqrt{\frac{2 N_{e} r_{p}}{L \sigma_{y}\left(\sigma_{x}+\sigma_{y}\right) A}} .
$$

Table 1 gives a list of ions and their corresponding frequencies for a $0.5-\mathrm{nC}$ beam. Fields from trapped ions provide extra focus on the electron beam and produce a shift of the betatron tune frequency. This tune shift is described by the following expression [2]:

$$
\Delta v_{y}=\frac{r_{e} \beta_{y} N_{e} \eta}{2 \pi \gamma \sigma_{y}\left(\sigma_{x}+\sigma_{y}\right)},
$$

where $r_{e}$ is the classical electron radius, $\beta_{\mathrm{y}}$ is the average vertical beta function, $\eta$ is the neutralization factor, and $\gamma$ is the relativistic energy of the electron beam. At full neutralization the tune shift for the PAR is estimated to be 0.02 , which is close to the time-captured beam spectrum observation.

Table 1: Ion Frequencies for a 0.5-nC Beam

\begin{tabular}{|c|c|}
\hline Mass of Ion, $\mathrm{A}$ & Frequency, $\mathrm{f}_{\mathrm{i}}(\mathrm{MHz})$ \\
\hline 2 & 0.888 \\
\hline 4 & 0.628 \\
\hline 16 & 0.314 \\
\hline 18 & 0.296 \\
\hline 28 & 0.237 \\
\hline 44 & 0.189 \\
\hline
\end{tabular}

Coherent oscillation occurs when ion and electron motions satisfy a resonance condition.

We theorize that in continuous injection mode, the newly injected beam has a larger beam size and is stable initially. Ion trapping happens when beam size is damped to a value that satisfies the condition for ion trapping, and the beam becomes unstable. As more ions are trapped into the beam, the ion-caused tune shift increases. The maximum tune shift is reached at full neutralization. Damping and bunch-length compression processes in the PAR cycle cause further changes in beam size, bunch length, and particle density. At some point the condition for ion trapping no longer exists and beam becomes stable again.

A similar argument can be made for the stored-beam mode case. In this case, beam has already damped to a small size, the ion trapping condition is not met, and beam is stable. Application of a kicker pulse excites the beam horizontally, which increase the beam size and decreases beam density. This produces a condition for ion trapping. Additional kicker-firing shakes off the trapped ion and causes the instability to stop.

\section{CHROMATICITY ADJUSTMENT}

The existence of this vertical instability occasionally causes problems when low charge injection is needed for certain SR bucket fill patterns.

Different tunings methods were explored in order to correct or weaken the instability. We tried ion clearing with the striplines and found it to be ineffective. That was probably because the locations of the striplines were not suited for ion cleaning. We found that increasing vertical chromaticity reduced the strength of the instability. As the chromaticity increased, the rate of oscillations seen on the synchrotron monitor decreased, and the beam spectra gradually returned to a stable condition. The instability disappeared when the vertical chromaticity was increased by 0.16 . This is probably due to the larger vertical tune spread, which makes the beam less coherent. The corrected lattice was optimized and installed for normal operations.

\section{CONCLUSION}

We have observed a vertical instability in the PAR. Investigations were performed to explore the frequency spectrum, and the spatial and timing pattern of this instability. A preliminary analysis concluded that this is a coherent ion trapping instability. As a result of the investigation, a more stable lattice was established.

\section{ACKNOWLEDGEMENTS}

The author would like to acknowledge many beneficial discussions and exchanges with Michael Borland, Lee Teng, Kathy Harkay and Alex Lumpkin on the subject.

\section{REFERENCES}

[1] David Sagan et al., "Ion trapping in the CESR BFactory," Proceedings of the 1991 PAC Conference, p. 1839, 1991.

[2] Y. Baconnier, "Neutralization of Accelerator Beams by Ionization of the Residual Gas," CERN-85-19, p. 267.

[3] David Sagan et al., "Observations of the coherent beam-ion interaction in the CESR storage ring," Nucl. Instrum. Methods, A 344 (1994) 459-469.

[4] P. Krejcik et al., "Ion Effects in the SLC Electron Damping Ring," Proceedings of the 1997 PAC Conference, p. 1626, 1997.

[5] S. Matsumoto et al., "Experimental study of the ion trapping phenomenon in Tristan-AR," Proceedings of the 1997 PAC Conference, p. 306, 1997. 